\title{
Analysis of Segmentation Performance on the CEDAR Benchmark Database
}

\author{
Michael Blumenstein and Brijesh Verma \\ School of Information Technology, Griffith University-Gold Coast Campus \\ PMB 50, Gold Coast Mail Centre, QLD 9726, Australia \\ E-mail: \{m.blumenstein, b.verma\}@mailbox.gu.edu.au
}

\begin{abstract}
The purpose of this paper is to analyse the performance of our improved segmentation algorithm tested on the CEDAR benchmark database. Segmentation is achieved through the extraction of a wide range of information adjacent to or surrounding suspicious segmentation points. Initially, a heuristic technique is employed to search for structural features and to over-segment each word. For each segmentation point that is located, the left character (preceding the segmentation point), and centre character (centred on the segmentation point) are extracted along with other features from the segmentation area. The aforementioned features are presented to trained character and segmentation point validation neural networks to evaluate a number of confidence values. Finally, the confidence values are fused to obtain the final segmentation decision. Based on a detailed analysis, it was observed that the left and centre character networks increased the accuracy of the segmentation algorithm.
\end{abstract}

\section{Introduction}

Researchers agree that segmentation plays an important role in the overall process of handwriting recognition [13]. There is still a need to improve segmentation accuracy and to compare results for the segmentation of handwriting using benchmark databases. Cursive word segmentation deserves particular attention as it has been acknowledged as the most difficult of all handwriting segmentation problems [4].

Few researchers have detailed their findings for the segmentation process of their handwriting recognition systems. Most researchers tend to measure the success of their system by their findings from the character or word recognition phases. In recent years, some researchers have developed measures for evaluating the performance of their segmentation systems $[5,6]$.

This research paper analyses the performance of a neural-based segmentation technique that employs three experts for validating segmentation points in over- segmented words. Each expert provides a confidence value based on a neural output. The neural confidences are then fused to provide an overall segmentation decision. The Left Character Confidence (LCC) and Centre Character Confidence (CCC) are obtained by passing relevant features to trained character networks. Finally, a Segmentation Point Validation (SPV) confidence is obtained after passing a segmentation area to a network trained with correct and incorrect segmentation point patterns.

Following experimentation, an analysis of LC, CC and SPV performance was conducted. It was observed that in most cases, the $\mathrm{LC}$ and $\mathrm{CC}$ networks increase performance of the segmentation algorithm. However, in some cases the LC and CC networks play a decisive role in making erroneous segmentation decisions.

The remainder of the paper is broken down into 4 sections. Section 2 describes the proposed, neural-based segmentation technique and Section 3 provides experimental results. An analysis of the results is performed in Section 4, and a conclusion is drawn in Section 5.

\section{Neural Confidence-based Segmentation Technique}

This section addresses the steps required to segment the handwritten words using the neural confidence-based segmentation technique.

\subsection{Brief Overview of the Segmentation Technique}

Step 1. Locate features (such as upper and lower word contours, holes, upper and lower contour minima, vertical density histograms, etc.) and over-segment the word using the heuristic, feature-based segmenter.

Step 2. Obtain confidence values from character and Segmentation Point Validation (SPV) neural networks. Step 3. Fuse all confidence values using the functions described in Section 2.4. 


\subsection{Heuristic Feature-based Over-segmenter}

The heuristic feature-based segmenter used in this paper locates Prospective Segmentation Points (PSPs) in handwritten words. It has been described in detail in [7].

\subsection{Neural Confidences}

Following heuristic segmentation it is necessary to discard "incorrect" segmentation points while preserving the "correct" points. This is achieved by calculating a number of confidences for each segmentation point generated by the heuristic segmenter. Three neural networks are used for this step. Firstly, a neural network is trained with features extracted from segmentation areas originally located by the heuristic algorithm. The neural network verifies whether each particular area is or is not characteristic of a segmentation point [7]. If an area is positively identified as a segmentation point, the network outputs a high confidence $(>0.5)$. Otherwise the network will output a confidence close to 0.1 .

Two other neural networks trained with handwritten characters (upper case and lower case) are then used to confirm the first neural network's output. Each network is presented with areas immediately centred on/adjacent to each segmentation point. Area width is calculated based upon average character width. If for example, the area immediately to the left of the PSP proves to be a valid character, the network will output a high confidence (LCC) for that character class. At the same time, if the area immediately centred on the segmentation point provides a high confidence for the reject neuron (CCC), then it is likely that the PSP is a valid segmentation point. The "reject" output is specifically trained to recognise noncharacter patterns (ie. joined characters, half characters or unintelligible primitives). If this neuron gives a high confidence, this will usually indicate that the particular area being tested is a good candidate for a segmentation point. Otherwise, if any valid characters are given a high confidence (in the centre character area), it is unlikely that that particular area should be segmented. Fusion of character and segmentation point confidences is detailed in the following section.

\subsection{Confidence Fusion}

A Correct Segmentation Point (CSP) is found:

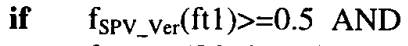

$$
\begin{aligned}
& \mathrm{f}_{\mathrm{LCC}} \text { Ver }(\mathrm{ft} 2) \text { is a high character confidence AND } \\
& \mathrm{f}_{\mathrm{CCC}-\mathrm{Ver}}(\mathrm{ft} 3) \text { is a high non-character confidence; }
\end{aligned}
$$$$
f_{C S P}(f t 1, f t 2, f t 3)=f_{S P V_{-} V e r}(f t 1)+f_{L_{C C} \text { Ver }}(f t 2)+f_{C_{C C} V_{e r}}(f t 3)
$$

where, $f_{S P V}$.Ver $($ features) - Confidence value from the Segmentation Point Validation neural network.

$\mathrm{f}_{\text {LCC_Ver }}$ (features) - Left Character Confidence (LCC) value from character neural network.

$\mathrm{f}_{\mathrm{CCC} \_ \text {Ver }}$ (features) - Centre Character Confidence (CCC) from character neural network (reject neuron output).

An Incorrect Segmentation Point (ISP) is found:

$$
\begin{aligned}
& \text { if } \quad \mathrm{f}_{\mathrm{SPV} V_{\mathrm{V}}}(\mathrm{ft} 1)<0.5 \mathrm{AND} \\
& \mathrm{f}_{\mathrm{LCC}} \mathrm{Ver}_{\mathrm{f}}(\mathrm{ft} 2) \text { is a high non-character confidence AND } \\
& \mathrm{f}_{\mathrm{CCC}-\mathrm{Ver}}(\mathrm{ft} 3) \text { is a high character confidence; }
\end{aligned}
$$

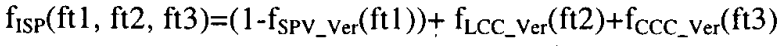

where, $f_{S P V}$ Ver $($ features) - Confidence value from Segmentation Point Validation neural network.

$f_{\text {LCC_Ver }}$ (features) - Left Character Confidence value from character neural network (reject neuron output).

$\mathrm{f}_{\mathrm{CCC}_{-} \text {ver }}$ (features) - Centre Character Confidence value from character neural network (highest confidence from 26 character neuron outputs)

Finally, the outcome of fusion is decided by the following equation:

$\mathrm{f}($ confidence $)=\max (\mathrm{f}(\mathrm{CSP}), \mathrm{f}(\mathrm{ISP}))$

\section{Experimental Results}

Samples of handwritten words from the CEDAR benchmark database [8] were used for training the SPV/character neural networks. In particular, all the words contained in the "BD/cities" directory of the CD-ROM were used. For testing, 300 words were obtained from the "BD/cities" test set.

Two main sets of segmentation experiments were conducted. The first set utilised heuristic oversegmentation followed by the SPV procedure on its own. The second set incorporated the fusion of SPV and character networks. The segmentation errors for both experiments are reported in terms of criteria used by Yanikoglu and Sandon [5] and Xiao and Leedham [6]. The three criteria used here are: 1) number of oversegmentations, 2) missed segmentations and 3) bad segmentations. "Over-segmentations" are obtained when a character is segmented into more than three pieces. "Missed" segmentations, refers to the number of correct segmentation points that were not located. Finally, "Bad" segmentations are those obtained when a segmentation point does not perfectly separate two characters. Instead, the point of segmentation (anchorage point or segmentation path) dissects a piece of an adjacent character or some similar anomaly.

For experiment one, all test words were segmented using the heuristic over-segmenter followed directly by the neural, SPV technique discussed in Section 2.3. Each 
segmentation point that was given a high confidence after validation was preserved, while those segmentation points that were given a low confidence were discarded. In addition, extra segmentation points were forced in areas of the word that were left un-segmented for an area greater than the average character width. The error rates may be seen below:

Table 1. Segmentation Errors for Experiment One

\begin{tabular}{|c|c|c|c|c|}
\hline & $\begin{array}{c}\text { Over- } \\
\text { Segmentation } \\
(\%)\end{array}$ & $\begin{array}{c}\text { Missed } \\
(\%)\end{array}$ & $\begin{array}{c}\text { Bad } \\
(\%)\end{array}$ & $\begin{array}{c}\text { Bad + } \\
\text { Correct } \\
\text { Anchorage } \\
\text { Points (\%) }\end{array}$ \\
\hline Errors: & $\mathbf{8 . 1 5}$ & 1.45 & 14.26 & 9.6 \\
\hline
\end{tabular}

For experiment two, the heuristic segmenter was once again employed to provide PSPs. Further to this, three confidence values were obtained for each point using the SPV network and the character networks examining left and centre character features. These confidences were then fused for each point and a decision of "correct" or "incorrect" was obtained. The results are tabulated below. Results for both experiments are compared in Figure 1.

Table 2. Segmentation Errors for Experiment Two

\begin{tabular}{|c|c|c|c|c|}
\hline & $\begin{array}{c}\text { Over- } \\
\text { Segmentation } \\
(\%)\end{array}$ & $\begin{array}{c}\text { Missed } \\
(\%)\end{array}$ & $\begin{array}{c}\text { Bad } \\
(\%)\end{array}$ & $\begin{array}{c}\text { Bad + } \\
\text { Correct } \\
\text { Anchorage } \\
\text { Points (\%) }\end{array}$ \\
\hline Errors: & 7.47 & 2.04 & 11.64 & 6.3 \\
\hline
\end{tabular}

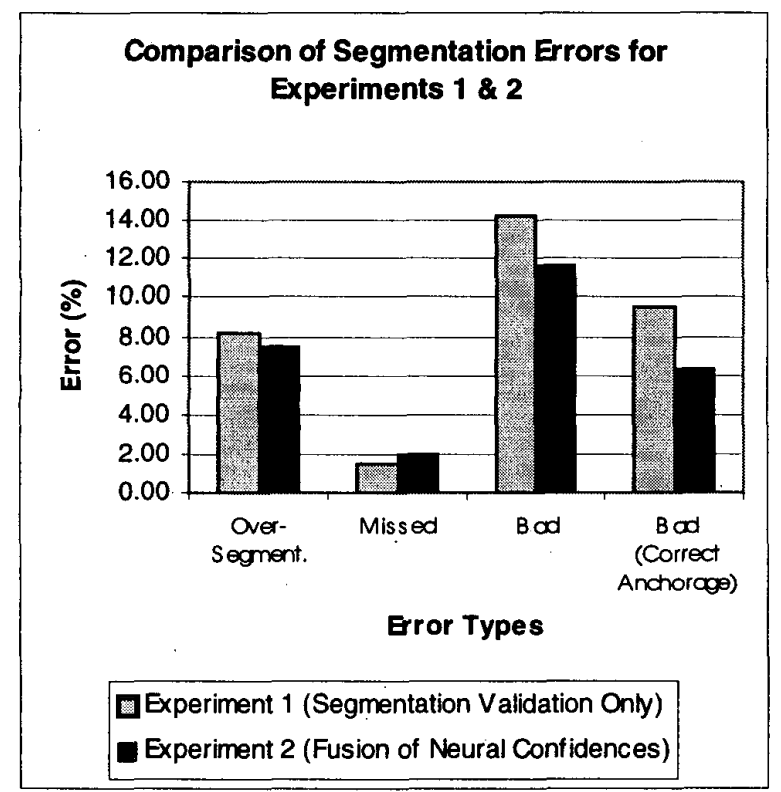

Figure 1. Comparison of Segmentation Errors for Experiments $1 \& 2$

\section{Analysis and Interpretation of Results}

\subsection{Comparison of Segmentation Results}

As may be seen from Table 1, the segmentation algorithm performed reasonably well. In particular the "missed" error was quite low at $1.45 \%$. The over-segmentation error was prominent, however not excessive. It should be noted that over-segmentation is not a decisive factor for correct word recognition in our system. However, it may be seen that a large number of "bad" segmentations were obtained $(14.26 \%)$. These were partly introduced due to the fact that the segmentation algorithm does not locate segmentation paths between characters, and therefore although the anchorage point is correct, the vertical segmentation may sometimes cut adjacent character components. The location of segmentation paths was considered beyond the scope of this research. Therefore if the "bad" segmentation error is expressed in terms of locating incorrect anchorage points only, it goes down significantly to: $9.6 \%$.

On comparing the results of the first experiment to those of the second, an improvement may be seen in the latter. The over-segmentation error drops by $0.68 \%$. However, at the same time, a slight increase for the number of missed segmentations also occurs (up 0.59\%). This slight variation may, in some cases, be attributed to incorrect extraction of character areas for network identification (discussed shortly). The change in error distribution may also be attributed to a lack of "weighting" when character confidences are fused. One final and quite positive observation may be made with respect to the "bad" segmentation error. It may be seen that it drops almost $3 \%$. It is also noted that when the "bad" segmentations are finally represented in terms of the number of correct anchorage points, a low error of $6.3 \%$ is obtained.

Figure 2 shows some examples of segmented words using SPV on its own (Experiment 1) and Segmentation with Neural Confidence Fusion (Experiment 2). For Experiment 1, it may be seen that in the word "Dallas", the "D" and "a" have not been correctly separated i.e. giving a "bad" segmentation. With character context and neural fusion, this problem is avoided in Experiment 2. Similar examples can be seen separating " $t$ "'s in the word "Seattle" and separating "I" and "N" in "Moines". All the above cases have correctly been dealt with using the fusion of neural confidences. Finally, an example where a "missed" segmentation has occurred for Experiment 1 may be seen in the word "maple". The PSP separating "l" and "e" has been totally removed using SPV only. However, with character context, the segmentation point has been correctly retained in Experiment 2. 


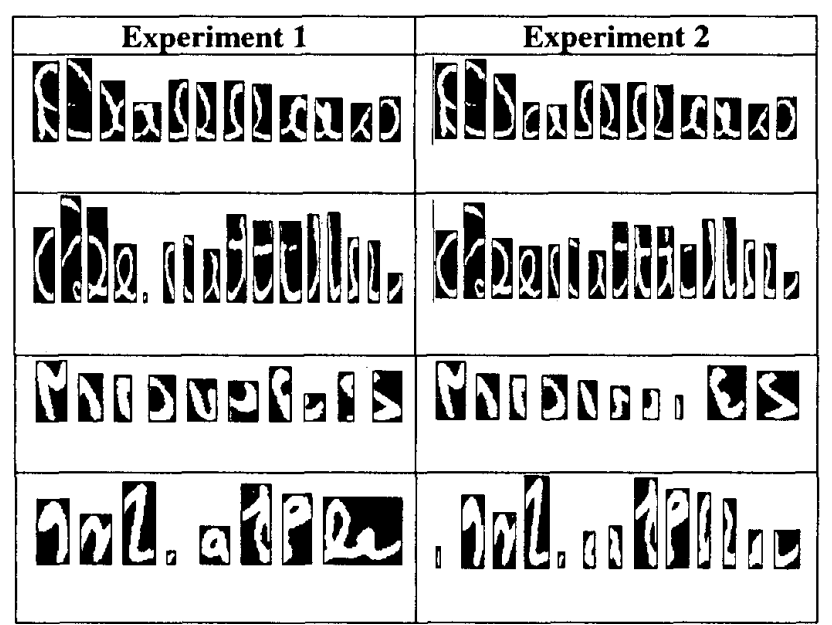

Figure 2. Examples of Segmented Handwritten Words for Experiments 1 and 2

For the second experiment, a number of errors were attributed to the calculation of character area size. For example, if the size of the area to be presented to the neural network is incorrectly calculated (average character width), then the area that is analysed may either be too small or too large. This may result in misclassification of a particular area as a character, and hence preserving an "incorrect" point.

In comparison with other researchers in the field, the results obtained in this research are comparable. For the results presented here, the "missed" rate is within approximately $1 \%$ of those found in [5] and [6]. Although the over-segmentation rate is higher in our research (by approximately $7 \%$ ), it must be stressed once again that over-segmentation is not a very important criterion for our system. Finally, bad segmentation (not including errors attributed to incorrect segmentation paths) is comparable within $4 \%$ and $4.5 \%$ of those obtained in [5] and [6] respectively. It may be noted that it is quite difficult to compare the results obtained here with those in $[5,6]$, as the authors used different data sets.

\subsection{Analysis of Individual Expert Performance}

An important component of this research was the performance analysis of individual experts (LCC, CCC and SPV) applied to the segmentation of handwritten words.

This section presents an analysis of expert performance on a sample of 80 words. The performance of experts was measured for successful segmentation outcomes i.e. correct segmentation points that were retained (C-R) and incorrect segmentation points that were deleted (I-D). As well as the above, the analysis also encompassed evaluating the performance of the experts when segmentation was unsuccessful in a word i.e. correct segmentations that were deleted (C-D) and incorrect segmentations that were retained (I-R). The tables and graphs below reflect the performance of individual experts on each of the cases mentioned above.

Table 3. Performance on C-R Segmentation Points

\begin{tabular}{|c|c|c|c|}
\hline & LCC & CCC & SPV \\
\hline \# of Correct Decisions & 350 & 288 & 459 \\
\hline \# of Incorrect Decisions & 115 & 205 & 34 \\
\hline \# of Non-Decisions & 28 & 0 & 0 \\
\hline
\end{tabular}

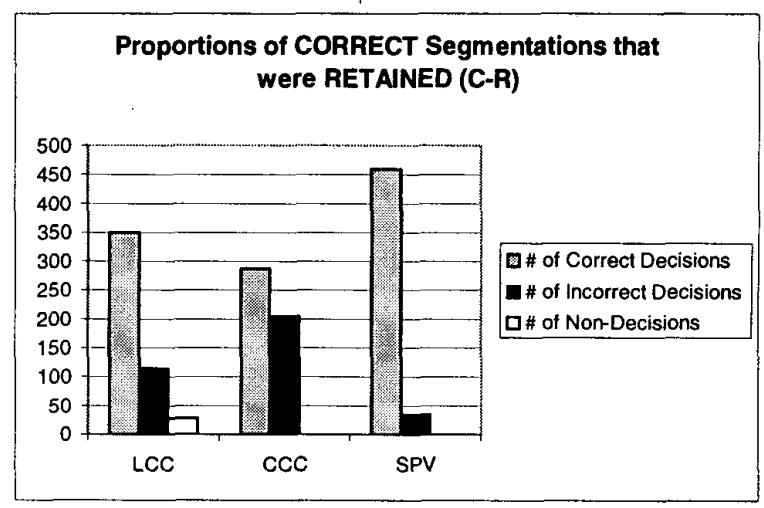

Figure 3. Visual Representation of Expert Performance on C-R Segmentation Points

Table 4. Performance on I-D Segmentation Points

\begin{tabular}{|c|c|c|c|}
\hline & LCC & CCC & SPV \\
\hline \# of Correct Decisions & 158 & 291 & 296 \\
\hline \# of Incorrect Decisions & 139 & 54 & 49 \\
\hline \# of Non-Decisions & 48 & 0 & 0 \\
\hline
\end{tabular}

Proportions of INCORRECT Segmentations that were DELETED (I-D)

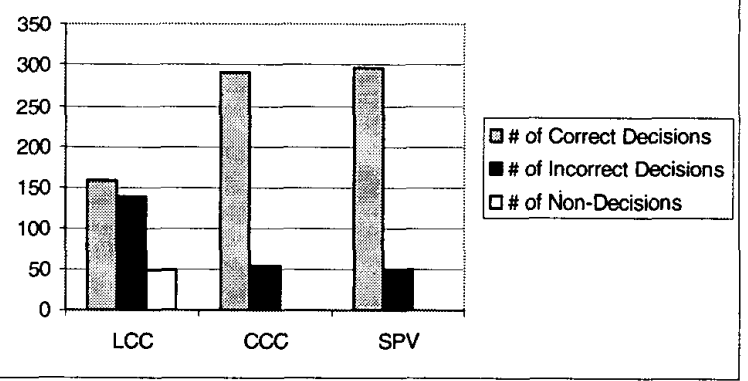

Figure 4. Visual Representation of Expert Performance on I-D Segmentation Points

Table 5. Performance on C-D Segmentation Points

\begin{tabular}{|c|c|c|c|}
\hline & LCC & CCC & SPV \\
\hline \# of Correct Decisions & 20 & 18 & 78 \\
\hline \# of Incorrect Decisions & 78 & 81 & 21 \\
\hline \# of Non-Decisions & 1 & 0 & 0 \\
\hline
\end{tabular}




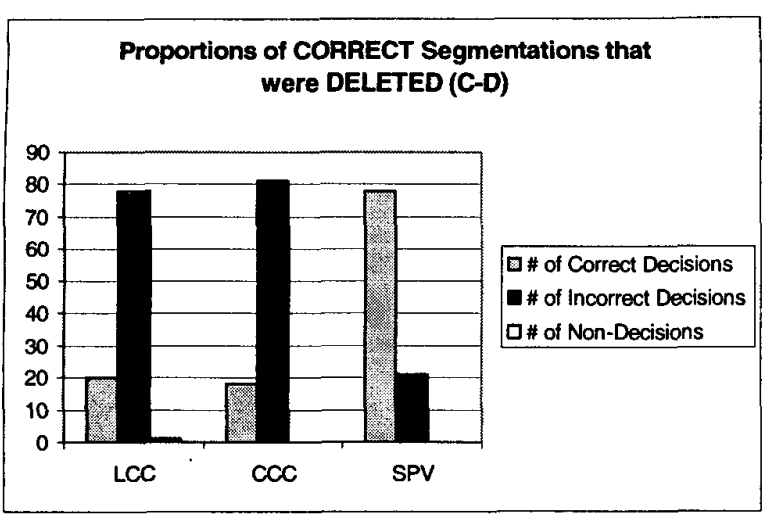

Figure 5. Visual Representation of Expert Performance on I-D Segmentation Points

Table 6. Performance on I-R Segmentation Points

\begin{tabular}{|c|c|c|c|}
\hline & LCC & CCC & SPV \\
\hline \# of Correct Decisions & 41 & 42 & 46 \\
\hline \# of Incorrect Decisions & 92 & 99 & 95 \\
\hline \# of Non-Decisions & 8 & 0 & 0 \\
\hline
\end{tabular}

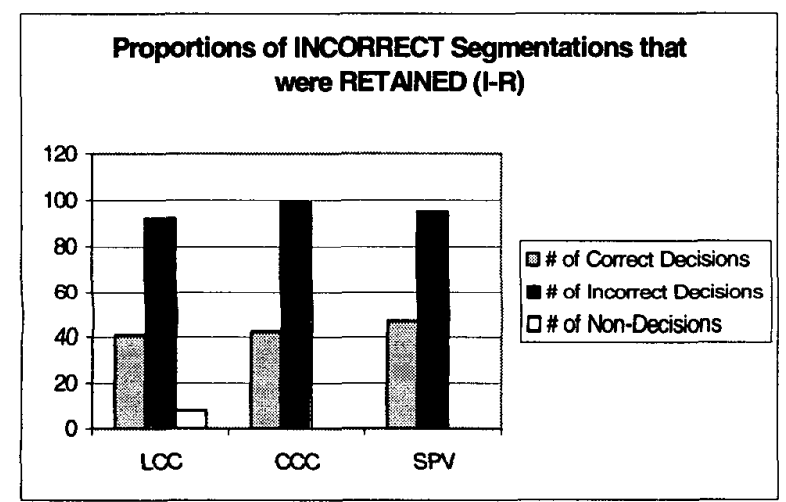

Figure 6. Visual Representation of Expert Performance on I-R Segmentation Points

A number of interesting observations can be made from the above tables and graphs. Firstly it may be observed that out of all the correctly retained/deleted segmentation points in Figures 3 and 4, the CCC and LCC respectively did not agree with the final segmentation decision in many instances. In these cases the neural recognisers were required to provide a high confidence for non-character patterns. It seems that the recognisers did not effectively carry out this decision. To deal with this problem in future research, the recognisers shall be re-trained with more exemplary features for non-character patterns. Other classifiers will also be tested.

It may also be observed from Figures 3 and 4 that the SPV network was consistent in providing correct decisions for the segmentation process. However, it may be noted that in the small number of instances that SPV failed, the LCC and CCC networks were able to assist by providing high confidences to invoke a correct decision.

Figure 5 clearly indicates that for those cases where a correct segmentation point was removed based on the combined confidence values, it was the LCC and CCC networks that contributed to most of the incorrect decisions. Conversely, Figure 6 shows that for the incorrect points that were mistakenly retained, all experts were equally to blame for providing an incorrect decision.

\section{Conclusion}

A detailed analysis of a fusion-based segmentation technique for cursive handwriting has been presented. The following observations were made based on the experiments conducted. Firstly, the left and centre character networks increase the accuracy of the segmentation algorithm. Further observations indicate that in a majority of cases the segmentation point validation network is quite accurate whereas the left and centre character networks incur difficulties when attempting to distinguish character and non-character patterns.

\section{References}

[1] S. N. Srihari, 1993, "Recognition of Handwritten and Machine-printed Text for Postal Address Interpretation", Pattern Recognition Letters, Vol. 14, pp. 291-302.

[2] R. G. Casey and E. Lecolinet, 1996, "A Survey of Methods and Strategies in Character Segmentation", IEEE Trans. PAMI, Vol. 18, pp. 690-706.

[3] M. Blumenstein and B. Verma, 1999, "Neural Solutions for the Segmentation and Recognition of Difficult Words from a Benchmark Database". ICDAR '99, Bangalore, India, pp. 281-284.

[4] Y. Lu and M. Shridhar, 1996, "Character Segmentation in Handwritten Words - An Overview", Pattern Recognition, Vol. 29, pp. 77-96.

[5] B. Yanikoglu and P. A. Sandon, 1998, "Segmentation of Off-Line Cursive Handwriting using Linear Programming", Pattern Recognition, Vol. 31, pp. 1825 1833.

[6] X. Xiao and G. Leedham, 2000, "Knowledge-based Cursive Script Segmentation", Pattern Recognition Letters, Vol. 21, pp. 945-954.

[7] M. Blumenstein and B. Verma, 1999, "A New Segmentation Algorithm for Handwritten Word Recognition”, IJCNN '99, Washington D.C., pp. 878-882.

[8] J. J. Hull, 1994, "A Database for Handwritten Text Recognition", IEEE Trans. PAMI, Vol. 16, pp. 550-554. 